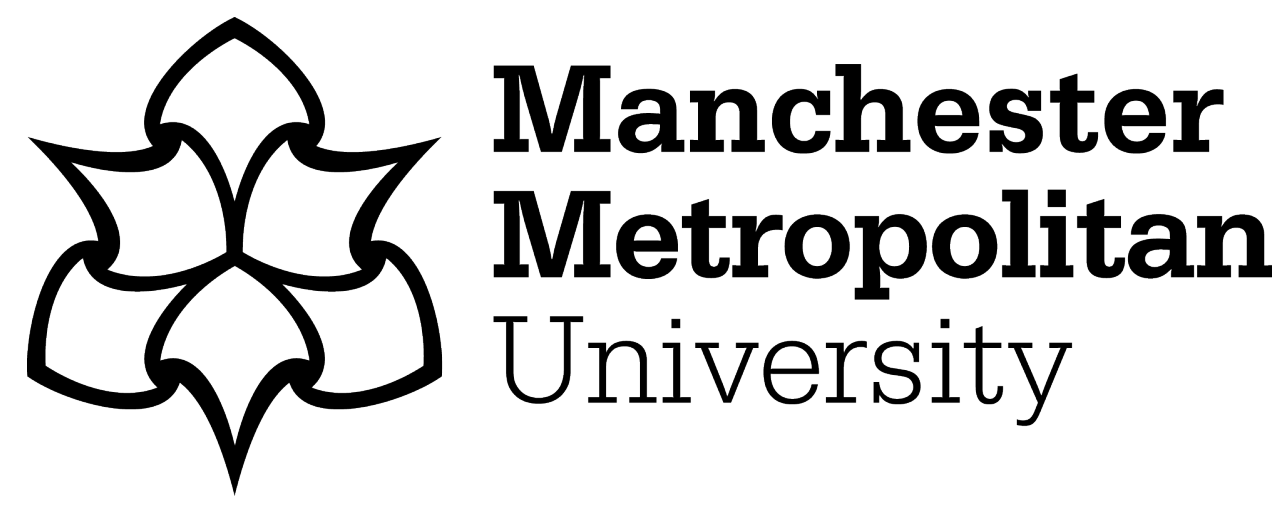

Deng, Zhuqing, Lloyd, Huw, Xia, Canwei, Møller, Anders, Liang, Wei and Zhang, Yanyum (2018) Components of variation in female common cuckoo calls. Behavioural Processes, 158. pp. 106-112. ISSN 0376-6357

Downloaded from: https://e-space.mmu.ac.uk/621632/

Version: Accepted Version

Publisher: Elsevier

DOI: https://doi.org/10.1016/j.beproc.2018.10.007

Usage rights: Creative Commons: Attribution-Noncommercial-No Derivative Works 4.0

Please cite the published version 
1 Title: Components of variation in female common cuckoo calls

2 Short running title: Female cuckoo calls

3 Authors: Zhuqing Deng ${ }^{1}$, Huw Lloyd ${ }^{2}$, Canwei Xia ${ }^{1 *}$, Anders Pape Møller ${ }^{1,3}$, Wei

$4 \quad$ Liang $^{4}$, Yanyun Zhang ${ }^{1 *}$.

5 1. Ministry of Education Key Laboratory for Biodiversity and Ecological Engineering,

6 College of Life Sciences, Beijing Normal University, Beijing 100875, China.

7 2. School of Science and Environment, Manchester Metropolitan University,

8 Manchester M1 5GD, UK.

9 3. Ecologie Systématique Evolution, Université Paris-Sud, CNRS, AgroParisTech, 10 Université Paris-Saclay, F-91405 Orsay Cedex, France

11 4. Ministry of Education Key Laboratory for Ecology of Tropical Islands, College of

12 Life Sciences, Hainan Normal University, Haikou 571158, China

13 * Corresponding author, Canwei Xia, Telephone: 0086-18901376801, ORCID: 0000-

14 0003-1432-1019; Yanyun Zhang, Telephone: 0086-13426164940

15 Email: Zhuqing Deng (dengzhuqing@mail.bnu.edu.cn)

16 Huw Lloyd (H.Lloyd@mmu.ac.uk)

17 Canwei Xia (xiacanwei@126.com)

18 Anders Pape Møller (anders.moller@u-psud.fr, ID: 0000-0003-3739-4675)

19 Wei Liang (liangwei@hainnu.edu.cn, ID: 0000-0002-0004-9707)

20 Yanyun Zhang (zhangyy@bnu.edu.cn)

21 Authors' contributions:

22 YZ, WL designed the experiments; ZD participated in the field work; ZD, CX carried

23 out the analyses; CX drafted the earlier version of the manuscript and HL, AM, ZD

24 revised it. All authors have read and approved the final manuscript.

25 Availability of data and materials: All recordings and data in this study are

26 available from the corresponding author (xiacanwei@126.com) on reasonable request.

27 Declarations of interest: none

28 Ethical standards: Our research protocol was approved by the Animal Management 
29 Committee at the College of Life Sciences, Beijing Normal University under license

30 number CLS-EAW-2016-017. Bird capture and banding were permitted by the

31 National Bird-banding Center of China under license number H20110042. The

32 experiments comply with the current laws of the country in which they were

33 performed.

34 


\section{Components of variation in female common cuckoo calls}

Highlights

Larger intra-individual variation in female than in male cuckoo calls

39 Peak frequency of female cuckoo call was significantly negative related to latitude

Cuckoos were more vocally active in sunny weather than rainy weather

The peak in vocal activity (both male and female cuckoos) was in the morning

Abstract: Investigations on bird vocalizations have largely focused on males. Female vocalizations are widespread in birds but few studies have investigated female vocal characteristics, particularly in non-Passeriformes. In this study, we use new field recordings from China, and calls available from an online sound library to examine temporal patterns, call consistency and geographical variation in vocalizations of female common cuckoos Cuculus canorous. The peak in vocal activity (both male and female) was in the morning, which contrasts to what would be predicted if the sole function of the female call was to distract the attention of hosts after parasitizing a host's nest in the afternoon. Both male and female common cuckoos were more vocally active in sunny weather, than rainy weather. We also found larger intra-individual variation in female rather than in male calls, which may benefit female cuckoos by increasing stimulation to host species. Peak frequency of female calls decreased with increased latitude, while differences in female call features were not associated with geographic distance. In summary, our findings that female calls are used in the morning, rather than at peak egg laying, yet are highly variable and show little geographic patterns suggest that the function of these female calls may be more variable and intricate than previously thought. However, because research on female vocalizations is underrepresented, future studies are still needed.

Key words: acoustic signals; call consistency; common cuckoo; female vocalization; 
geographical variation; vocal activity.

\section{Introduction}

Studies of bird vocalizations have been critical in shaping our understanding of the relationship between avian signal evolution and species differentiation (Andersson, 1994). Historically, most attention on variation in avian acoustic signals has been directed towards males (Beecher and Brenowitz, 2005), with many bird populations showing regional differences in male vocal characteristics over local and/or wider geographical scales (Irwin, 2000; Boughman, 2002; Kaluthota et al., 2016). However, female vocalizations are also widespread in both temperate and tropical species (Garamszegi et al., 2007; Yang et al., 2011; Odom et al., 2014; Mahr et al., 2016).

Whilst females of some species use their acoustic signals to defend territotries, coordinate breeding activities and attract mates (Langmore, 1998). Mate attraction across bird species does not appear to be the primary function of female vocalizations (Dabelsteen et al., 1998; Cain and Langmore, 2016; Krieg and Getty, 2016). Female vocalisations vary in their complexity (Langmore, 1998; Cain and Langmore, 2016) but few studies have examined whether female vocal characteristics vary geographically, particularly for non-Passeriformes (Odom and Benedict, 2018). Given the prevalence of female vocalizations, more research should be conducted to build a comprehensive understanding of the function and evolution of bird vocalizations. Male common cuckoos Cuculus canorous utter loud simple and stereotypic "cuck-ooo" calls during the breeding season. Temporal and frequency variables among different male cuckoo calls are sufficient to provide individual information (Jung et al., 2014; Li et al., 2017; Zsebok et al., 2017), which can be used to distinguish between neighbors and strangers (Moskat et al., 2017). Furthermore, there appears to be a high degree of consistency in the number of syllables in each bout produced by individual males (Møller et al., 2016a, b). However, males of different subspecies differ significantly in their calls (Wei et al., 2015), even within the same 
91 subspecies, male calls from populations in the same habitat are more similar to each 92 other than those from populations in different habitats (Fuisz and de Kort, 2007). In comparison, our knowledge of the characteristics and function of female cuckoo calls

94 have, until recently, been largely overlooked. Female cuckoos often give a conspicuous 'chuckle' call (Payne, 2005). One recent experimental playback study suggests that the chuckle call primarily serves as a distraction of the host parent species (e.g. reed warbler Acrocephalus scirpaceus) since female chuckle calls had the same effect on the attention of host and non-host species, as playback of the calls of sparrowhawk Accipiter nisus (York and Davies, 2017). In contrast, male common cuckoo calls had no such effect. Such a function would enable female cuckoos to

101 benefit from reducing their egg rejection rate through distraction of the attention of

102 hosts (York and Davies, 2017). Other aspects of female cuckoo calls remain

103 unquantified in the peer-review literature (Liang, 2017; Kim et al., 2017). In this study, we use new recordings and those deposited in the online avian acoustic sound library Xeno-Canto, to examine temporal patterns, individual consistency, and geographical variation in the chuckle calls of female common cuckoos. Unlike most birds, which lay egg in the morning, female cuckoos

108 predominantly lay their eggs in the afternoon (Payne, 2005), and the function of

109 female cuckoo calls is to distract the host species after laying (York and Davies, 110 2017). Therefore, we predicted that peak female cuckoo calls should occur during the 111 afternoon. Since sound signals are more easily distorted in bad weather (e.g. rain or 112 strong winds) than good weather (e.g. sunny) (Lengagne and Slater, 2002), we 113 predicted that cuckoo calls would be more frequent on days with good weather. 114 Theoretically, elaborate signals could increase stimulation of sensory perception 115 (Akre and Johnsen, 2014; Cui et al., 2016), which may reduce habituation in the 116 distraction of the attention cuckoo hosts. Consequently, we predicted that female calls 117 should be more variable than male calls. Besides the potential difference in call 118 characteristics among different subspecies and populations in different habitats, there 
may also be differences in female call characteristics among different host races

120 within the same population. As female cuckoo calls functionally mimic hawk calls

121 (York and Davies, 2017), different populations may exhibit different call

122 characteristics due to different geographic variation in hawk calls and/or hawk species

123 with different calls occurring within the resident bird community. Thus, our final

124 objective was to determine whether female chuckle calls exhibit geographic variation,

125 but because this could be more complex than those for males, we made no specific

126 prediction about geographic variation in female cuckoo calls.

\section{Methods}

\subsection{Study area and sound recording}

130 Fieldwork was conducted during June $8^{\text {th }}$ to July $28^{\text {th }} 2017$ in the Liaohe Delta Nature

131 Reserve $\left(41.034^{\circ} \mathrm{N} ; 121.725^{\circ} \mathrm{E}\right)$, Liaoning Province, Northeast China. This region

132 represents one of the most important estuarine wetlands in China, with the largest area

133 of reed-bed habitat along the coastal region of China, and consequently, extensive

134 nesting habitats for Oriental reed warbler Acrocephalus orientalis. Here, the common

135 cuckoo is a summer breeding species, and it predominantly parasitizes Oriental reed

136 warbler nests (Li et al., 2016). Density of cuckoos is high in our study population (Li

137 et al. 2016), where several individuals often occur in close proximity (less than $10 \mathrm{~m}$ )

138 to each other but data on territory size is lacking. We used a TASCAM DR-100MKIII

139 recorder (Tascam Co., Japan) and a Sennheiser MKH416 P48 external directional

140 microphone (Sennheiser Co., Germany), with a sampling rate of $44.1 \mathrm{kHz}$ and a

141 sampling accuracy of 16 bits, to record cuckoo vocalizations. Further recordings were

142 made using seven passive acoustic recorder SM4 Songmeters (Wildlife Acoustics Inc.

143 USA) placed at seven different locations, separated by a minimal distance of $200 \mathrm{~m}$,

144 to continuously record cuckoo calls from June $8^{\text {th }}$ to July $28^{\text {th }}$. The minimal distance

145 between these recorders is larger than the effective recording range (100 $\mathrm{m}$ for

146 cuckoos call, seen in Huang et al., 2017), in order to avoid the same call being 
recorded by two recorders. Recorders were attached to telegraph poles at a height of 3 $\mathrm{m}$ above ground, and set to record continuously at a sampling rate of $44.1 \mathrm{kHz}$, and a sampling accuracy of 16 bits. Recorders were checked every 10 days to replace the batteries and memory cards. Using mist nets, we also trapped and banded 20 individual adult common cuckoos (6 females and 14 males) around our recording sites, whilst daily observations also revealed many other unbanded individual cuckoos at our recording sites during data collection.

To examine geographic variation in female cuckoo song, we downloaded all recordings of female common cuckoo from the online sound library Xeno-Canto (http://www.xeno-canto.org). For multiple recordings collected on the same day at the same location such that individuals could not be identified, we randomly selected one recording for analysis to avoid pseudo-replication. We also used four recordings that we collected from Liaohe Delta Nature Reserve, and four recordings collected from Beijing Wild Duck Lake National Wetland Park $\left(40.410^{\circ} \mathrm{N} ; 115.829^{\circ} \mathrm{E}\right)$, which is situated approximately $500 \mathrm{~km}$ from the Liaohe Delta Nature Reserve. These eight recordings were made in June 2017 with the same equipment mentioned before.

\subsection{Acoustic measurements}

All recordings were re-sampling with $8 \mathrm{kHz}$, and saved as .wav files. We used Avisoft-SASLab Pro 5.2 audio analysis software (Avisoft Bioacoustics, Germany) to generate spectrograms with the following settings: Fast Fourier transform length 256 points; Hamming window with a frame size of $100 \%$ and an overlap of $50 \%$; frequency resolution $31 \mathrm{~Hz}$; and time resolution of $16 \mathrm{~ms}$. Here the phrase 'number of calls' refers to number of syllables in males, and the number of calling bouts in females. Male cuckoo calls consist of a repeated series of ' $c u-c o o$ ' syllables (Møller et al., 2016a; Li et al., 2017) i.e. both 'cu' and 'coo' elements constitute a single syllable with several successive syllables constituting a bout. The pause between successive male calling bouts was always longer than $2 \mathrm{~s}$ in our recordings, which is obviously 
greater than the time interval between successive ' $\mathrm{cu-coo'} \mathrm{syllables} \mathrm{within} \mathrm{one} \mathrm{calling}$ bout (Fig. 1a). Female cuckoo calls consist of a series of rapidly repeated " $k w i k-k w i k$ kwik" notes (York and Davies, 2017), which we named a bout (Fig. 1b). Each "kwik" represented a syllable, corresponding to the terminology used for male calls. For each bout (both male and female), we measured maximum frequency, minimum frequency, peak frequency, duration, and the number of syllables. Peak frequency refers to the frequency associated with the maximum energy. Setting a standard to measure minimum frequency was problematic because energy decreases gradually towards low frequency in female cuckoo calls. Consequently, we did not include minimum frequency in the subsequent analyses.

To describe the temporal patterns of male and female cuckoo vocal activity, we used Kaleidoscope Pro Software (Wildlife Acoustics Inc. USA) to create recognizers for finding all male and female calls from recordings collected with the SM4 Song meters. Firstly, we entered the following acoustic features of our target sound, either a male syllable or female bout, to Kaleidoscope Pro Software: frequency range from 400 to $1200 \mathrm{~Hz}$ for a male syllable, and 600 to $2900 \mathrm{~Hz}$ for a female calling bout; duration ranges from 0.3 to $0.5 \mathrm{~s}$ for a male syllable, and 1.6 to $4 \mathrm{~s}$ for a female calling bout. These acoustic features are slightly larger than actual parameters of male and female cuckoo calls, but this was done to increase the detectability of these calls by the Kaleidoscope Pro Software. Lastly, we manually checked all calls identified by the recognizer based on visual inspection of the spectrograms. In total, we obtained 701,661 male syllables, and 2407 female bouts after manual check.

\subsection{Temporal patterns of vocal activity}

We used both our field recordings and recordings downloaded from Xeno-Canto to examine temporal patterns of vocal behavior by male and female cuckoos. Using our field recordings from June $8^{\text {th }}$ to July $24^{\text {th }}$ we first calculated the number of calls per hour using absolute time since we lacked data on twilight time. Our prediction was 
203 that female cuckoos had a peak call output in the afternoon, based on the primary

204 function of female calls being the distraction of hosts during egg laying by cuckoos.

205 Thus, we assumed that the use of absolute time, rather than twilight time, would have

206 little or no effect on tests of our prediction. We used a generalized linear mixed model

207 (GLMM) to estimate the repeatability of number of calls per hour, based on the

208 function $r p t$ in the R package $r p t R$ (Stoffel et al., 2017). After confirming that most

209 variation occurred among hours, rather than during the same hour on different days

210 (repeatability of number of calls per hour in male $0.763 \pm 0.056$ mean $\pm \mathrm{SE} ; 95 \%$

211 confidence interval ranged from 0.623 to $0.828 ; P<0.001$; in female $=0.872 \pm 0.087$

212 mean $\pm \mathrm{SE} ; 95 \%$ confidence interval ranged from 0.609 to $0.910 ; P<0.001$ ), we

213 pooled the data by calculating the mean number of calls per hour, and we used these

214 data to illustrate temporal patterns of cuckoo vocal activity.

215 For a second data source, we used the time of cuckoo calls from the recordings

216 downloaded from Xeno-Canto, again using absolute rather than twilight time for the

217 analysis. From this second source, we obtained the time of 359 recordings of male

218 calls, and 36 recordings of female calls and calculated the number of recordings for

219 every two-hour period. Pearson correlation coefficient based on the number of calls

220 (or recordings) in each hour from the Liaohe Delta Nature Reserve recordings and

221 those from Xeno-Canto were used to determine patterns of similarity in vocal activity

222 from the two separate data sources. Finally, we related patterns of vocal activity from

223 the first data source to localized weather conditions. We defined weather condition

224 based on the information from Weather China (www.weather.com.cn), and classified a

225 day as a bad i.e. days with rain or strong winds (with wind speed greater than $8 \mathrm{~m} / \mathrm{s}$ ) or

226 good i.e. days without rain and strong winds.

$228 \quad 2.4$ Individual call consistency

229 We used a TASCAM DR-100MKIII recorder and a Sennheiser MKH416 P48 external

230 directional microphone to obtain recordings from 18 males in the Liaohe Delta Nature 
231 Reserve during June and July 2017; six of these males were color-banded from our

232 mist-netting efforts, whilst further recordings were made from unbanded males from

233 locations separated by at least $2 \mathrm{~km}$ from each other, in an effort to reduce the

234 likelihood of repeatedly sampling the same individual twice. Since female calls were

235 much rarer than male calls in our study area (Fig. 2), we supplemented the dataset

236 with recordings made using the passive acoustic recorder SM4 Songmeters. Since we

237 could not be certain which individual calls belonged to the same individual in passive

238 acoustic recordings, we defined two bouts recorded by the same recorders within 1

239 min and with similar amplitude as being from the same individual because we found

240 that it is rare to hear different female calls at the same time in adjacent sites. Using

241 this definition, we obtained 43 recordings, each containing two bouts, from 6

242 songmeters. These recordings were recorded in different locations, or at the same

243 location on different days. Since common cuckoos are abundant in our study area (Li

244 et al., 2017) we treated these 43 recordings as being derived from 43 different female

245 individuals. To avoid pseudo-replication, we used a smaller data set only including six

246 recordings (corresponding to six individual females) recorded from different

247 locations, randomly selected from the original 43 recordings. We calculated the

248 Pearson correlation coefficient from measurements on two successive bouts, and used

249 this to reflect individual call consistency. We compared the correlation coefficients

250 between males and females in the larger data set $(n=43)$, using the Fisher $\mathrm{z}$ -

251 transformation to assess the significance of the difference between two correlation

252 coefficients. We did not calculate the significance of the difference between

253 correlation coefficients between males and females in the smaller data set because of

254 the small sample size in the smaller data set $(n=6)$.

\subsection{Geographical variation}

257 We measured 35 female recordings from Xeno-Canto, and supplemented this dataset

258 with four recordings from Liaohe Delta Nature Reserve, and four recordings from 
Wild Duck Lake National Wetland Park. Although different equipment was used to

260 collect recordings by Xeno-Canto recorders, we assume that any effects of equipment

261 on recordings should only cause noise in the data set, and there is no reason to expect

262 bias in such effects. We restricted our analyses to one bout in each recording, as most

263 recordings downloaded from Xeno-Canto only contained one bout. Since acoustic

264 measures vary by different orders of magnitude e.g. the frequency of cuckoo syllables

265 ranges in the hundreds of $\mathrm{Hz}$, while the duration of syllables lasts nearly a tenth of a

266 second, acoustic measures were transformed into z-scores, and then used to calculate

267 the Euclidean distance of all pairs of bouts. We used Mantel tests to assess the

268 correlation between Euclidean distance and geographic distance and Pearson

269 correlation to compared the acoustic measures with latitude, as acoustic

270 characteristics are known to change with latitude in many bird species (Kaluthota et

271 al., 2016; Wei et al., 2017). All statistical analyses were performed using R software,

272 v. 3.4.1 (R Core Development Team, 2017). Data are presented as mean \pm standard

273 deviation. Differences with $P$ values less than 0.05 were considered significant.

\section{3. Results}

2763.1 Temporal variation in common cuckoo calls

277 At the Liaohe Delta Nature Reserve, we found that the number of cuckoo calls per

278 day fluctuated widely, with a sharp decline after July $25^{\text {th }}$ (Fig. 2). The number of

279 male and female cuckoo calls per day was significantly positively correlated (Pearson

280 correlation, $\mathrm{r}=0.075, P<0.001, \mathrm{n}=47$ ) and both male and female common cuckoos

281 showed higher vocal activity during good weather (Fig. 3). Males on average uttered

$28217,192 \pm 1,241($ mean \pm SD) syllables summarized from data collected by seven

283 recorders per day in good weather $(n=25$ days), significantly more than the $11,867 \pm$

284899 syllables per day during bad weather $\left(\mathrm{n}=22\right.$ days; independent samples $\mathrm{t}$ test, $t_{45}$

$285=3.392, P=0.001)$. Females uttered $61 \pm 6$ bouts summarized from data collected by

286 seven recorders per day in good weather $(n=25$ days), significantly larger than $40 \pm 7$ 
287 bouts per day during bad weather ( $\mathrm{n}=22$ days; independent samples $\mathrm{t}$ test, $t_{45}=2.406$, $288 P=0.02$ ). Data from both the Liaohe Delta Nature Reserve (Fig. 4a) and Xeno-Canto 289 (Fig. 4b) revealed that peak call output occurred during the morning rather than in the 290 afternoon, with noticeably little female call activity in the afternoon at the Liaohe 291 Delta Nature Reserve. Number of calls per hour from Liaohe Delta Nature Reserve 292 and Xeno-Canto were strongly positively correlated in both males $(\mathrm{r}=0.769, P<$ $2930.001)$ and females $(\mathrm{r}=0.655, P=0.001)$.

\subsection{Individual consistency}

296 Males $(n=18)$ generally showed higher individual call consistency than females $(n=$

297 43) (Fig. 5). Pearson correlation coefficients of maximum frequency (male: $r=0.926$;

298 female: $r=0.618$ ), duration (male: $r=0.488$; female: $r=0.067$ ), and number of 299 syllables (male: $r=0.609$; female: $r=0.087$ ) were significantly larger for males than 300 for females ( $Z$ test, $Z=3,1.98,2.05 ; P=0.003,0.048,0.040$, respectively). Pearson 301 correlation coefficients of peak frequency were larger in females $(r=0.523)$ than in 302 males $(\mathrm{r}=0.179)$, but the difference was not significant ( $Z$ test, $Z=1.32, P=0.187$ ).

303 Pearson's correlation coefficients for females in the smaller data set (6 recordings 304 made from different locations) showed a similar trend as Pearson's correlation 305 coefficients for females in the larger data set (43 recordings made from different 306 locations, or at the same location on different days): If Pearson correlation coefficients 307 for females in the larger data set was larger (or lower) than Pearson correlation 308 coefficients for males, Pearson's correlation coefficients for females in the smaller 309 data set was also larger (or lower) than in males for each variable (Fig. 5).

\subsection{Geographical variation}

312 We found in female calls a maximum frequency of bouts of 2,228 $\pm 40 \mathrm{~Hz}$; peak

313 frequency of bouts of $1,905 \pm 47 \mathrm{~Hz}$; duration of bouts of $1.979 \pm 0.056 \mathrm{~s}$, with 19.53 $314 \pm 0.78$ syllables. There was no significant linear trend of differences in female calls 
315 and geographic distance (Mantel test, $P=0.256$ ) (Fig. 6a). Peak frequency decreased 316 with increased latitude (Pearson's correlation coefficients, $\mathrm{r}=-0.364, P=0.016, \mathrm{n}=$

317 43) (Fig. 6b), while maximum frequency $(\mathrm{r}=-0.166, P=0.289)$, duration $(\mathrm{r}=-0.031$, $318 P=0.842)$, and number of syllables $(\mathrm{r}=-0.094, P=0.547)$ showed no significantly 319 relationship with latitude.

\section{Discussion}

\subsection{Temporal patterns of female vocal activity}

323 Based on the field recordings from Liaohe Delta Nature Reserve and others deposited on the online sound library Xeno-Canto, we found that vocal activity of female cuckoos peaked in morning rather than in the afternoon. We admit that the call recordings in Xeno-Canto are influenced by human activities (Blackburn et al., 2014) e.g. observers are more likely to spend more time recording birds in good weather and in the morning, which may lead to bias in the interpretation of daily vocal behavior patterns. In addition, to avoid potential bias due to differences in time across the different time zones represented in the Xeno-Canto recordings, we used the regional local time rather than twilight time as this would have little or no effect on tests of our

332 prediction of peak call output in the afternoon. We used automatic recognizers 333 generated from our field recordings to automatically identify potential cuckoo calls, 334 all of which were then subsequently checked manually by visual inspection of the spectrograms. Previous research on Large Hawk Cuckoo (Hierococcyx sparverioides)

336 found that only about $50 \%$ of all cuckoos calls were correctly identified by automatic 337 recognizers from 96 hours of recordings (Huang et al., 2017). Most of the common 338 cuckoo calls that were not detected by the automatic recognizers in this study 339 occurred during the dawn chorus, and were largely masked by the dawn songs of 340 oscine passerines. Thus, female cuckoos may have higher peak of call output in the 341 morning than we observed.

342 The peak of female call output in the morning is in contrast to what would be 
343 predicted if the only function of the female cuckoo call was to distract the attention of

344 hosts after parasitizing a host's nest i.e. peak in call activity should occur during the

345 afternoon (see York and Davies, 2017). Perhaps, female calls may be used to find

346 nests of hosts in the morning. As most birds in the morning lay eggs or sit on eggs,

347 female cuckoos produce calls which cause that host leave the nest, and then locate

348 nests of hosts. It is also likely that female common cuckoo calls at our study site have

349 other functions besides distraction hosts (Liang, 2017). For example, females calling

350 in the morning may attract the attention of males (e.g. Langmore, 1998).

351 Alternatively, it may be the case that potential host species at Liaohe Delta Nature

352 Reserve are able to distinguish between female common cuckoo calls from hawk

353 calls, or even use female calls as a predictor of risk of parasitism or increased

354 probability of egg rejection, although further experimental playback research is

355 needed.

4.2 Individual call consistency

358 Individual consistency as measured by repeatability is important in social behavior,

359 but also as an upper limit for heritability and hence micro-evolution (Bell et al. 2009;

360 Nakagawa and Schielzeth 2010). Male common cuckoo call features are consistent

361 within individuals (Jung et al., 2014; Li et al., 2017; Zsebok et al., 2017), which can

362 facilitate neighbor - stranger discrimination in birds (Moskat et al., 2017). For

363 females, York and Davies (2017) reported that the primary function of calls is to

364 distract the attention of host species. Elaborate signals could increase stimulation of

365 sensory perception (Akre and Johnsen, 2014; Cui et al., 2016), which may reduce

366 habituation of distraction of the attention of hosts. In agreement with this prediction,

367 females generally show lower individual consistency in call characteristics than

368 males: specifically, Pearson's correlation coefficients of maximum frequency,

369 duration, and number of syllables in females are significantly lower than those of

370 males. These results were consistent across both the larger (43 recordings recorded in 
371 different locations, or at the same location on different days) and the smaller (6

372 recordings recorded in different locations) data sets thus we believe that our sample

373 sizes were sufficient to evaluate both within- and between-individual differences.

374 Pearson's correlation coefficients of peak frequency in female calls was larger than in

375 males, although the difference was not significant. For many bird species, peak

376 frequency is determined by body size (Fletcher, 2004; Rodriguez et al., 2015) and is 377 consistently higher in male cuckoo calls (Jung et al., 2014; Li et al., 2017; Zsebok et

378 al., 2017). This acoustic feature could potentially be used for monitoring female 379 cuckoos.

\subsection{Geographical variation in female cuckoo calls}

382 Geographic variation in bird vocalizations is common, and may affect mate choice, 383 pair bonding, and territory defense in both passerine species such as stonechat 384 Saxicola torquata (Mortega et al., 2014) and coal tit Periparus ater (Pentzold et al. 385 2016), and also non-passerine species such as gentoo penguin Pygoscelis papua 386 (Lynch and Lynch, 2017) and corncrake Crex crex (Budka and Osiejuk, 2017). One of 387 the more common patterns in bird acoustic geographic variation can be described in 388 terms of the 'isolation by distance' model (Podos and Warren, 2007), in which 389 vocalization differences in paired populations increase with the distance between 390 those populations (Irwin, 2000; Xing et al., 2013). This pattern can be caused by both 391 cultural drift and genetic differences, as there are few chances for culture and genetic 392 exchange among remote populations (Irwin et al., 2008; Stewart and MacDougall393 Shackleton, 2008; Ramsay and Otter, 2015). In accordance with this model, 394 differences in calls and geographic distance are known to be closely correlated in 395 male common cuckoos (Wei et al., 2015). However, in our study we found no

396 evidence of isolation by distance, and we found no significant linear trend in 397 differences in female cuckoo calls with geographic distance. For female cuckoos, 398 there may be differences in call characteristics among different races within the same 
population, besides potential differences in call characteristics among different subspecies and populations in different habitats. If the purpose of female cuckoo calls

401 is to functionally mimic hawk calls (York and Davies, 2017), different populations

402 may have different call features due to different hawk species with different calls

403 occurring in the local bird community. These factors could lead to complexity in 404 geographic variation of female cuckoo calls, and mask any linear trend between 405 differences in calls and geographic distance as predicted in the isolation by distance 406 model.

407 Peak frequency is thought to correlate negatively with body size (Fletcher, 2004; 408 Rodriguez et al., 2015) but it is not unusual to find evidence to the contrary to this 409 rule in some oscines (e.g. rufous-collared sparrow Zonotrichia capensis Handford and 410 Lougheed 1991; dark-eyed junco Junco hyemalis and serin Serinus serinus Cardoso et 411 al. 2008), due to song learning and sexual selection (Patel et al., 2010; Cardoso, 412 2012). However, this rule is generally supported in suboscines and non-passerines, 413 particularly for species with large body size and low acoustic frequency in 414 vocalizations such as doves (Tubaro and Mahler, 1998), tinamous (Bertelli and 415 Tubaro, 2002), and antbirds (Seddon, 2005). In this study, we found that peak 416 frequency of female cuckoo calls decreased at higher latitude. In cuckoos, both male 417 and female body size is known to increase at higher latitudes (Payne, 2005; Erritzøe et 418 al., 2012), and this may lead to the negative relationship between peak frequency and 419 latitude.

\section{Conclusions}

422 In this study, we investigated three aspects (temporal patterns, call consistency, 423 geographical variation) concerning female common cuckoo calls. York and Davies 424 (2017) suggested that female common cuckoos mimic sparrowhawk vocalizations in 425 order to distract the host species after laying. In accordance with this prediction, we found larger intra-individual variation in female calls, which may increase stimulation 
of the host species. Besides, we found peak frequency of female calls decreased with increased latitude, while differences in female call features were not associated with geographic distance. However, the daily vocal pattern contrasts with what would be predicted according to York and Davies (2017). If the only function of the female common cuckoo call was to distract the attention of hosts after parasitizing a host's nest, peak call output should occur during the afternoon because female common cuckoos predominantly lay their eggs in the afternoon (Payne, 2005). Data from both the Liaohe Delta Nature Reserve and Xeno-Canto revealed that peak call output occurred during the morning rather than in the afternoon. Based on this result, we infer that female common cuckoo calls have other functions besides distraction hosts.

\section{Acknowledgements}

We thank Xiaomeng Zhao, Boning Xue, Qi Wang, Qi Luo, Qingbin Wang, Shuang Guan, Ziqiang Huang, Linyu Jin and Juan Chen for assistance in the field work.

\section{Funding}

This study was supported by the National Key Technology R \& D Program of China (No. 2016YFC0503200), State Forestry Administration of China (No. KJHX2016135) and the National Natural Science Foundation of China (No. J1210075 and No. 31872243 to YZ, No. 31601868 to CX, and 31472453 to WL).

\section{References}

Akre KL, Johnsen S, 2014. Psychophysics and the evolution of behavior. Trends Ecol. Evol. 29: 291-300.

Andersson M, 1994. Sexual selection. Princeton: Princeton University Press.

Beecher MD, Brenowitz EA, 2005. Functional aspects of song learning in songbirds. Trends Ecol Evol 20: 143-149.

Bell AM, Hankison SJ, Laskowski KL, 2009. The repeatability of behaviour: a metaanalysis. Anim Behav 77: 771-783.

\section{Bertelli S, Tubaro PL, 2002. Body mass and habitat correlates of song structure in a} primitive group of birds. Biol J Linn Soc 77: 423-430. 
Blackburn TM, Su S, Cassey P, 2014. A Potential metric of the attractiveness of bird song to humans. Ethology 120: 305-312.

Boughman JW, 2002. How sensory drive can promote speciation. Trends Ecol Evol 17: $571-577$.

Budka M, Osiejuk TS, 2017. Microgeographic call variation in a non-learning species, the Corncrake (Crex crex). J Ornithol 158: 651-658.

Cain KE, Langmore NE, 2016. Female song and aggression show contrasting relationships to reproductive success when habitat quality differs. Behav Ecol Sociobiol 70: 1867 - 1877.

Cardoso GC, 2012. Paradoxical calls: the opposite signaling role of sound frequency across bird species. Behav Ecol 23: 237-241.

Cardoso GC, Mamede AT, Atwell JW, Mota PG, Ketterson ED et al., 2008. Song frequency does not reflect differences in body size among males in two oscine species. Ethology 114: 1084-1093.

Cui J, Song X, Zhu B, Fang G, Tang Y et al., 2016. Receiver discriminability drives the evolution of complex sexual signals by sexual selection. Evolution 70: 922-927.

Dabelsteen T, McGregor PK, Lampe H, Langmore N, Holland J, 1998. Quiet song in song birds: an overlooked phenomenon. Bioacoustics 9: 89-105.

Erritzøe J, Mann CF, Brammer FP, Fuller RA, 2012. Cuckoos of the World. London: Christopher Helm.

Fletcher NH, 2004. A simple frequency-scaling rule for animal communication. $J$ Acoust Soc Am 115: 2334-2338.

Fuisz TI, de Kort SR, 2007. Habitat-dependent call divergence in the common cuckoo: is it a potential signal for assortative mating? Proc $R$ Soc Lond B 274: 2093-2097.

Garamszegi LZ, Pavlova DZ, Eens M, Møller AP, 2007. The evolution of song in female birds in Europe. Behav Ecol 18: 86-96.

Handford P, Lougheed SC, 1991. Variation in duration and frequency characters in the song of the rufous-collared sparrow, Zonotrichia capensis, with respect to habitat, trill dialects and body size. Condor 93: 644-658.

Huang W, Xu S, Liang W, Xia C, 2017. Daily vocal pattern of large hawk cuckoo (Hierococcyx sparverioides). Chinese J Zool 52: 945-953.

Irwin D, 2000. Song variation in an avian ring species. Evolution 54: 998-1010.

Irwin DE, Thimgan MP, Irwin JH, 2008. Call divergence is correlated with geographic and genetic distance in greenish warblers (Phylloscopus trochiloides): a strong role for stochasticity in signal evolution? J Evolution Biol 21: 435-448.

Jung WJ, Lee JW, Yoo JC, 2014. "cu-coo": Can you recognize my stepparents? A study of host-specific male call divergence in the common cuckoo. PLoS ONE 9: e90468.

Kaluthota C, Brinkman BE, Dos Santos EB, Rendall D, 2016. Transcontinental 
latitudinal variation in song performance and complexity in house wrens (Troglodytes aedon). Proc R Soc Lond B 283: 20152765.

Kim H, Lee JW, Yoo JC, 2017. Comparing vocal structures of the parasitic and nonparasitic groups in Cuculinae. Avian Res 8: 27.

Krieg CA, Getty T, 2016. Not just for males: females use song against male and female rivals in a temperate zone songbird. Anim Behav 113: 39-47.

Langmore NE, 1998. Functions of duet and solo songs of female birds. Trends Ecol Evol 13: 136-140.

Lengagne T, Slater PJB, 2002. The effects of rain on acoustic communication: tawny owls have good reason for calling less in wet weather. Proc R Soc Lond B 269: 2121-2125.

Li D, Ruan Y, Wang Y, Chang AK, Wan D et al., 2016 Egg-spot matching in common cuckoo parasitism of the oriental reed warbler: effects of host nest availability and egg rejection. Avian Res 7: 199-209

Li Y, Xia C, Lloyd H, Li D, Zhang Y, 2017 Identification of vocal individuality in male cuckoos using different analytical techniques. Avian Res 8: 21

Liang W, 2017. Crafty cuckoo calls. Nat Ecol Evol 1: 1427-1428.

Lynch MA, Lynch HJ, 2017. Variation in the ecstatic display call of the gentoo penguin (Pygoscelis papua) across regional geographic scales. Auk 134: 894902.

Møller AP, Morelli F, Mousseau TA, Tryjanowski P, 2016a. The number of syllables in Chernobyl cuckoo calls reliably indicate habitat, soil and radiation levels. Ecol Indic 66: 592-597.

Møller AP, Morelli F, Tryjanowski P, 2016b. Cuckoo folklore and human well-being: cuckoo calls predict how long farmers live. Ecol Indic 72: 766-768.

Mahr K, Seifert CL, Hoi H, 2016. Female and male blue tits (Cyanistes caeruleus) sing in response to experimental predator exposition. J Ornithol 157: 907-911.

Mortega KG, Flinks H, Helm B, 2014. Behavioural response of a migratory songbird to geographic variation in song and morphology. Front Zool 11: 85.

Moskat C, Elek Z, Ban M, Geltsch N, Hauber ME, 2017. Can common cuckoos discriminate between neighbours and strangers by their calls? Anim Behav 126: $253-260$.

Nakagawa S, Schielzeth H, 2010. Repeatability for Gaussian and non-Gaussian data: a practical guide for biologists. Biol Rev 85: 935-956.

Odom KJ, Benedict L, 2018. A call to document female bird songs: applications for diverse fields. Auk 135: 314-325.

Odom KJ, Hall ML, Riebel K, Omland KE, Langmore NE, 2014. Female song is widespread and ancestral in songbirds. Nat Commun 5: 3379-3379.

Patel R, Mulder RA, Cardoso GC, 2010. What makes vocalisation frequency an unreliable signal of body size in birds? A study on black swans. Ethology 116: 554-563.

Payne RB, 2005. The Cuckoos. Oxford: Oxford University Press. 
Pentzold S, Foerschler MI, Tietze DT, Randler C, Martens J et al., 2016. Geographic variation in coal tit song across continents and reduced species recognition between central European and Mediterranean populations. Vertebr Zool 66: 191-199.

Podos J, Warren PS, 2007. The evolution of geographic variation in birdsong. $A d v$ Study Behav 37: 403-458.

Ramsay SM, Otter KA, 2015. Geographic variation in white-throated sparrow song may arise through cultural drift. J Ornithol 156: 763-773.

Rodriguez RL, Araya-Salas M, Gray DA, Reichert MS, Symes LB et al., 2015. How acoustic signals scale with individual body size: common trends across diverse taxa. Behav Ecol 26: 168-177.

Seddon N, 2005. Ecological adaptation and species recognition drives vocal evolution in neotropical suboscine birds. Evolution 59: 200-215.

Stewart KA, MacDougall-Shackleton EA, 2008. Local song elements indicate local genotypes and predict physiological condition in song sparrows Melospiza melodia. Biol Lett 4: 240-242.

Stoffel MA, Nakagawa S, Schielzeth H, 2017. rptR: repeatability estimation and variance decomposition by generalized linear mixed-effects models. Methods Ecol Evol 8: 1639-1644.

Tubaro PL, Mahler B, 1998. Acoustic frequencies and body mass in new world doves. Condor 100: 54-61.

Wei C, Jia C, Dong L, Wang D, Xia C et al., 2015 Geographic variation in the calls of the common cuckoo (Cuculus canorus): isolation by distance and divergence among subspecies. J Ornithol 156: 533-542

Wei C, Price TD, Liu J, Alström P, Zhang Y, 2017. The evolutionary origin of variation in song length and frequency in the avian family Cettiidae. $J$ Avian Biol. 48: 1295-1300.

Xing X, Alström P, Yang X, Lei F, 2013. Recent northward range expansion promotes song evolution in a passerine bird, the light-vented bulbul. J Evolution Biol 26: 867-877.

Yang C, Zhang Y, Cai Y, Stokke BG, Liang W, 2011. Female crowing and differential responses to simulated conspecific intrusion in male and female Hainan partridge (Arborophila ardens). Zool Sci 28: 249-253.

York JE, Davies NB, 2017. Female cuckoo calls misdirect host defences towards the wrong enemy. Nat Ecol Evol 1: 1520-1525.

Zsebok S, Moskat C, Ban M, 2017. Individually distinctive vocalization in common cuckoos (Cuculus canorus). J Ornithol 158: 213-222.

\section{Figure captions:}

Figure 1. Spectrogram of male (A), and female (B) common cuckoo calls, recorded in Liaohe Delta Nature Reserve $\left(41.034^{\circ} \mathrm{N} ; 121.725^{\circ} \mathrm{E}\right)$. 
585 Figure 2. The number of calls by male and female common cuckoos per day during the 2017 breeding season, summarized from data collected by seven recorders.

Figure 3. Call output (number of syllables summarized from data collected by seven recorders per day $\pm \mathrm{SE}$ ) by (A) male and (B) female common cuckoos in relation to different weather conditions. Asterisk indicates significant difference at $P<0.05$ (independent samples t test).

Figure 4. Daily temporal pattern of common cuckoo call (A) based on recordings from seven recorders in Liaohe Delta Nature Reserve; (B) 359 male recordings and 36 female recordings from the online sound library XenoCanto. Error bars are SE.

Figure 5. Pearson correlation coefficients from measurements of vocalization features in common cuckoos in two successive bouts. Asterisk indicates significant difference at $P<0.05$ ( $\mathrm{Z}$ test). $\mathrm{n}$ is the number of individuals and error bars are SE.

602

Figure 6. (A) Lack of significant linear trend in female common cuckoo calls with geographic distance (Mantel test, $P=0.256$ ); and (B) significant negative correlation between peak frequency and latitude (Pearson's correlation coefficients, $\mathrm{r}=-0.364, P=0.016, \mathrm{n}=43$ ). 

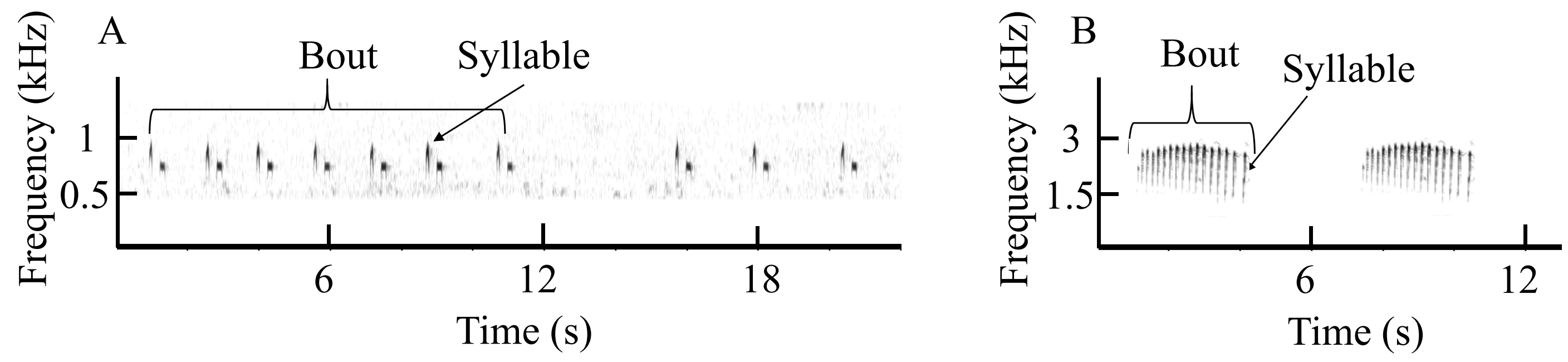


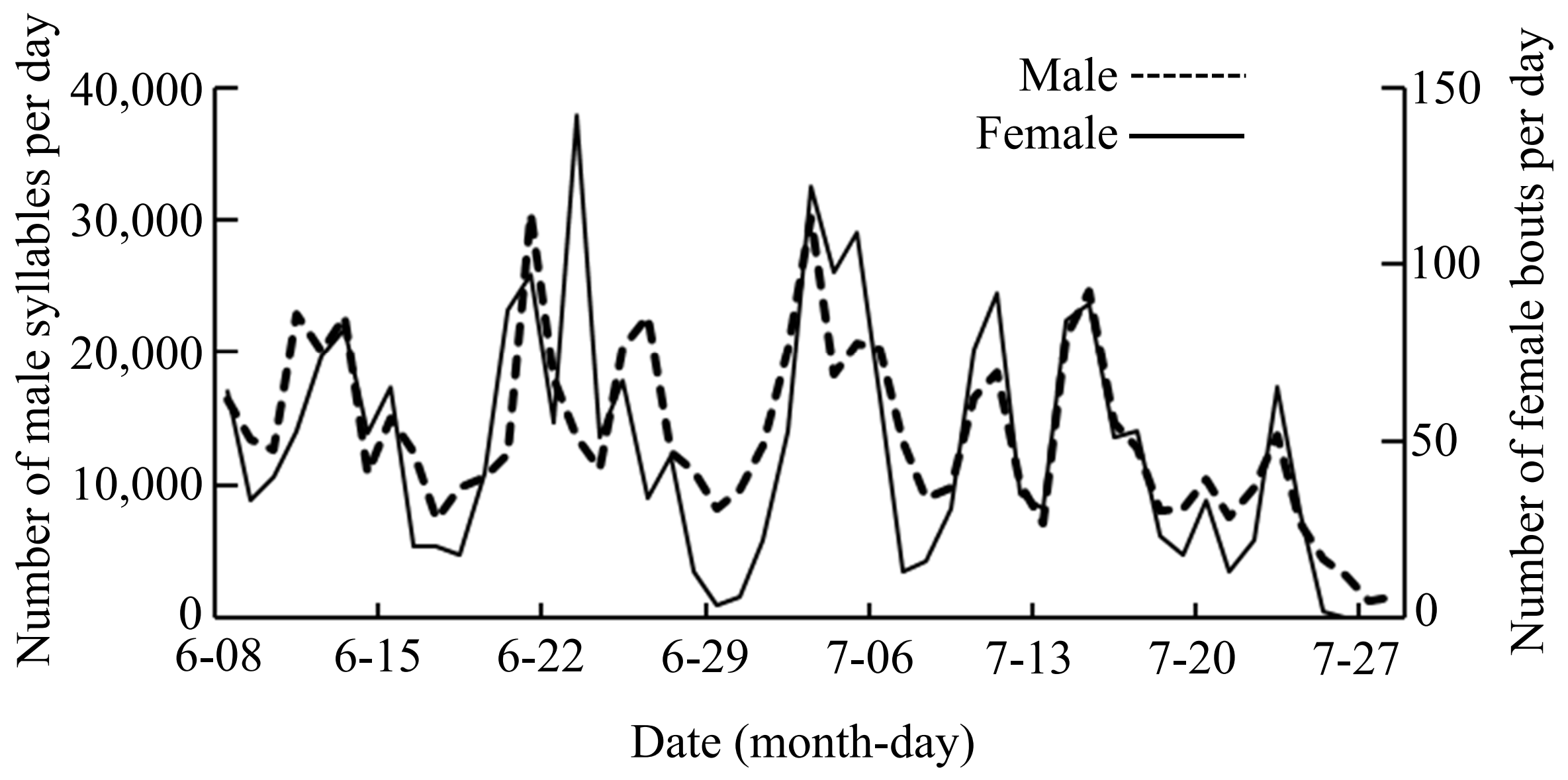




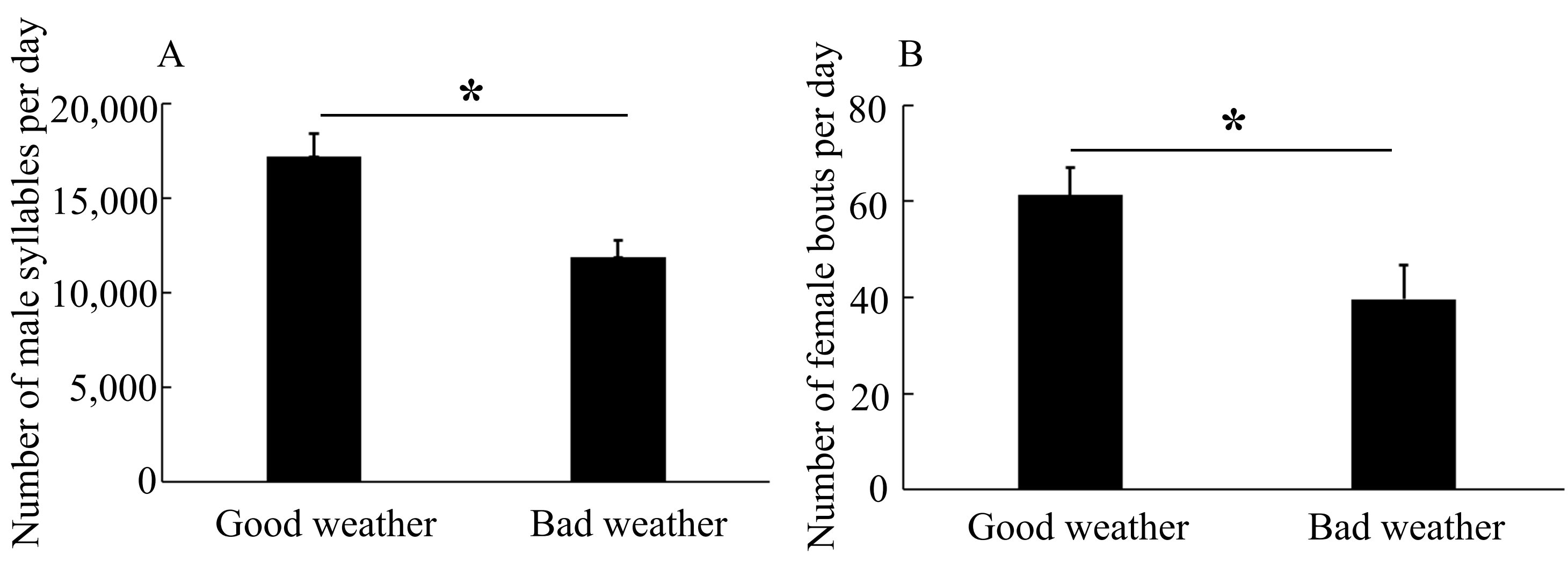



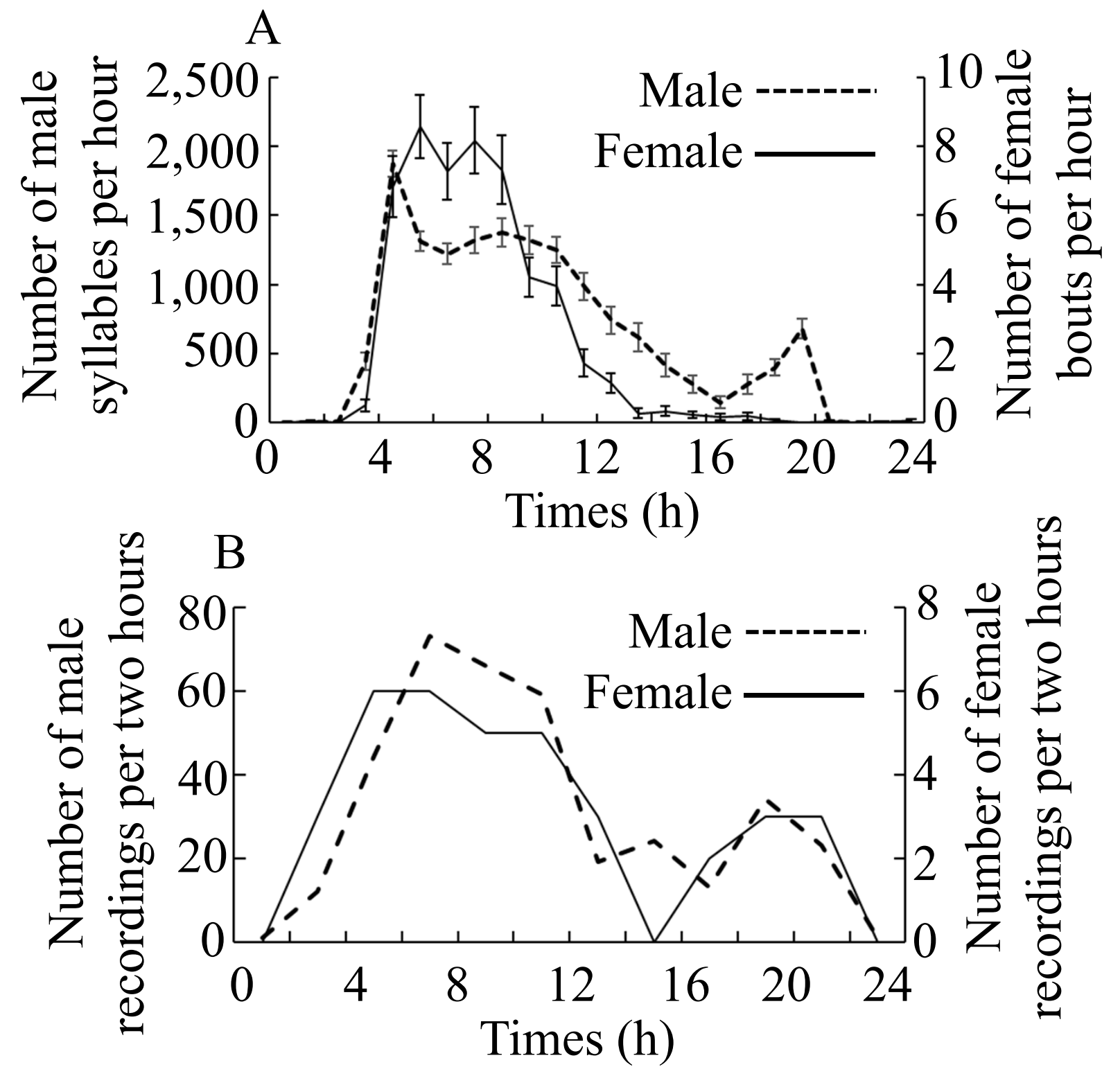


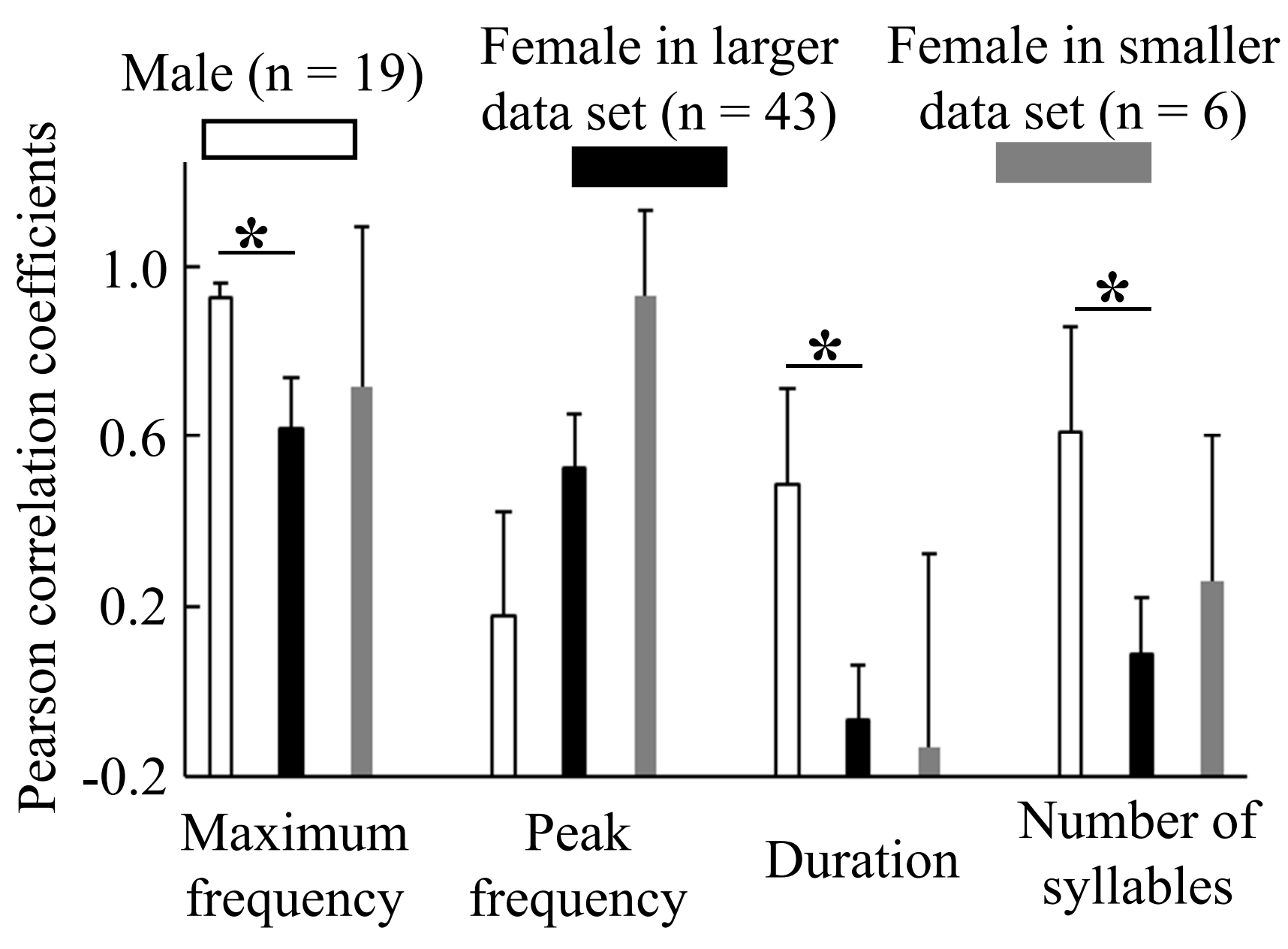



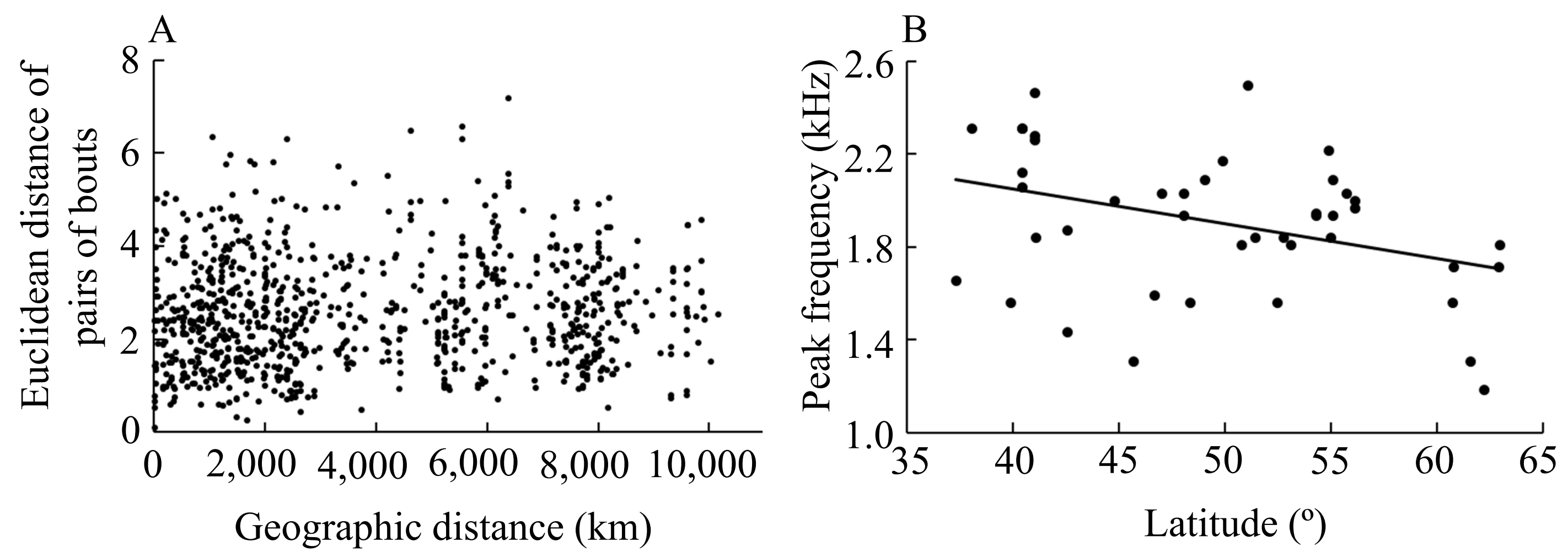